\title{
COHOMOLOGICAL INVARIANTS OF COMPLEX MANIFOLDS COMING FROM EXTREMAL RAYS*
}

\author{
JAROSLAW A. WIŚNIEWSKI ${ }^{\dagger}$
}

Introduction: good rays and bad rays. Topology has always been a very convenient tool for studying compact complex manifolds. In particular, the cohomology ring $H^{*}(X, \mathbf{Z})$ of a compact complex manifold $X$ is a very important invariant which provides a lot of information on the geometry of $X$. In the present paper we use $H^{*}(X, \mathbf{Z})$ to understand extremal ray contractions in the sense of the Minimal Model Program or Mori Theory.

Originally, an extremal ray contraction was invented to serve as a step in the Minimal Model Program on the way to reach a minimal or canonical model of a projective variety. However, it was soon observed that such contractions can measure certain properties of non-general varieties. Recently the notion of an extremal ray and its contraction has made its way to other branches of geometry: non-projective geometry, Moishezon manifolds, deformation of complex structure and symplectic geometry. At this point I should note that in this paper I use the name "extremal ray" (and respectively "extremal contraction") for both Mori (or Fano-Mori) and crepant extremal rays, see the definitions in Section 2.

It is apparent from some of these applications of extremal rays that not all rays can be equally treated. For example, in symplectic geometry [20] (c.f. [18]) we distinguish the rays which contain classes of good rational curves with good deformation properties.

In the present paper I try to explain this apparent non-equality of extremal contractions on the level of cohomology. Namely, using the invariants of $H^{*}(X, \mathbf{R})$ which come from the Hard Lefschetz Theorem I divide extremal rays in two classes: $L$ supported and L-negligible (where "L" stands for "Lefschetz"). Roughly speaking: L-supported rays are strongly distinguishable in topology while L-negligible rays have very mild geometry. Now the decision which rays are good and which are bad depends on the taste, or rather on a possible purpose they should serve for.

Let me summarize the properties of these two classes of rays. Each L-supported ray $R$ defines a hyperplane $R^{\perp} \subset H^{2}(X, \mathbf{R})$ on which Lefschetz duality degenerates (i.e. $R^{\perp}$ is a component of the Lefschetz discriminant defined in Section 1), so the hyperplane is a cohomology ring invariant. The hyperplane $R^{\perp}$ carries a multiplicity $m(R)$ (a cohomology ring invariant) which is related to the geometry of the ray $R$, for example $m(R) \geq l(R)-1$ where $l(R)$ is the length of the ray $R$ defined in [22]. The number of L-supported rays is finite and it can be bounded (counting them with their multiplicities) by the number $\sum_{k=1}^{n} k \cdot b_{n-k}(X)$.

Although the number of L-negligible rays may be infinite and they are invisible in the cohomology ring, their geometry is easier than that of L-supported rays. For example, in dimension 2 and 3 the only Mori extremal rays come from blowing up a smooth variety along a codimension 2 smooth center. Further, in codimension 4 contractions of Mori extremal rays can be well described (Theorem 3.4). Moreover,

\footnotetext{
*Received March 13, 1998; accepted for publication July 10, 1998. Dedicated to Matgosia, Jagna and Maryña.

†Instytut Matematyki Uniwersytetu Warszawskiego, Banacha 2, 02-097 Warszawa, Poland (jarekw@mimuw.edu.pl).
} 
each L-negligible ray contains lots of "good" rational curves whose deformation is of the expected dimension. In effect, L-negligible rays are invariant under deformations of complex structure and can be used to compute Gromov-Witten invariants in symplectic geometry.

The paper is organised as follows. After reviewing basic definitions concerning topology and Mori Theory, in Section 1 some consequences of Hard Lefschetz Theorem are worked out. In particular, the notions of Lefschetz discriminant and L-supported homology class are introduced. In Section 2, after recalling some further facts from Mori Theory concerning extremal contractions a representability theorem is proved; it allows to represent cohomology in the hyperplane $R^{\perp} \subset H^{2}(X, \mathbf{R})$ by cohomology of the target of the contraction of $R$. The geometry of L-negligible rays is studied in Section 3.

This paper was motivated by some recent publications on extremal rays in different branches of geometry. Apart of [20] and [18] I should mention also [21], [19] and [2]. I would like to thank the authors of the last two papers for their kind sending me their preprints. Moreover, I would like to thank the referee of the present paper who has brought to my attention the section 12.1 of [14] which contains results related to my Section 2. In some sense the present paper is a sequel of [24] where the idea of using Hard Lefschetz Theorem in the context of Mori Theory was born.

During the prepation of this paper I had a fellowship at the Institute of Mathematics of Polish Academy of Sciences. In April and May 1997 I lectured on the topology of complex manifolds at the University of Trento where I presented the results of this paper. I am very grateful to the participants of these lectures, especially to Marco Andreatta, Edoardo Ballico and Massimiliano Mella, for their remarks and criticism which corrected some of my wrongthinking. I was also partially supported by Polish KBN. I would like to thank all the above institutions for their kind support.

Notation and definitions. Throughout the present paper $X$ is a complex manifold of complex dimension $n$ and moreover - with the exception of a local set-up in Section $2-X$ is compact or just projective. We consider the complex topology of $X$. We will usually work with homology and cohomology of $X$ with coefficients in the field of real numbers $\mathbf{R}$; a cycle (or cocycle) with real coefficients will be called integral or rational if it is coming from homology (or cohomology) with integral or, respectively, rational coefficients.

A great deal of information about the relation of the complex structure of $X$ with its topology is carried by the exponential sequence on $X$ :

$$
0 \rightarrow \mathbf{Z}_{X} \longrightarrow \mathcal{O}_{X} \stackrel{\exp (2 \pi i \cdot)}{\longrightarrow} \mathcal{O}_{X}^{*} \rightarrow 0
$$

the long cohomology sequence of which defines the link between the Picard group of $X$ and its second cohomology group and in particular the first Chern class map:

$$
\begin{aligned}
& H^{1}\left(X, \mathbf{Z}_{X}\right) \longrightarrow H^{1}\left(X, \mathcal{O}_{X}\right) \longrightarrow P i c X \\
= & H^{1}\left(X, \mathcal{O}_{X}^{*}\right) \stackrel{c_{1}}{\longrightarrow} H^{2}\left(X, \mathbf{Z}_{X}\right) \longrightarrow H^{2}\left(X, \mathcal{O}_{X}\right)
\end{aligned}
$$

The image of $c_{1}$ in the torsion-free cohomology $H^{2}(X, \mathbf{Z}) /($ torsion) coincides with the group of divisors modulo numerical equivalence $\operatorname{PicX} / \equiv$.

Let $N^{1}(X) \subset H^{2}(X, \mathbf{R})$ and $N_{1}(X) \subset H_{1}(X, \mathbf{R})$ be $\mathbf{R}$-linear subspaces spanned by, respectively, cohomology and homology classes of, respectively, holomorphic divisors and curves on $X$. In other words $N^{1}(X)$ is spanned by the image of the map 
$c_{1}: \operatorname{Pic} X \rightarrow H^{2}(X, \mathbf{Z})$ composed with the extension of coefficients $H^{2}(X, \mathbf{Z}) \rightarrow$ $H^{2}(X, \mathbf{R})$.

The topological intersection of cycles and cocycles restricts to $N_{1}(X)$ and $N^{1}(X)$ and coincides with the intersection product which can be defined in algebro-geometric set up; in both cases the intersection of a cocycle $\chi$ with a cycle $\alpha$ will be denoted by $\chi \cdot \alpha$. The intersection product gives a non-degenerate pairing on $H^{2}(X, \mathbf{R}) \times H_{2}(X, \mathbf{R})$ and $N^{1}(X) \times N_{1}(X)$ and thus we will frequently identify any space in question with the dual of its pairing partner. For a given non-zero cycle $\alpha \in H_{2}(X, \mathbf{R})$ we define the perpendicular hyperplane $\alpha^{\perp}:=\left\{\chi \in H^{2}(X, \mathbf{R}): \chi \cdot \alpha=0\right\}$; similarly we define the hyperplane perpendicular to a non-zero cocycle.

Inside the above spaces we consider the following cones. The cone of curves $\mathcal{C} \subset N_{1}(X)$ and the cone of nef divisors $\mathcal{P} \subset N^{1}(X)$ are $\mathbf{R}_{>0}^{*}$-spanned on, respectively, the classes of curves and numerically effective divisors, i.e. $\mathcal{P}:=\left\{\chi \in N^{1}(X): \forall \alpha \in\right.$ $\mathcal{C} \quad \chi \cdot \alpha \geq 0\}$. That is $\mathcal{P}=\mathcal{C}^{\vee}$ in the sense of the intersection pairing of $N^{1}(X)$ and $N_{1}(X)$. Dually, $\overline{\mathcal{C}}=\mathcal{P}^{\vee}$, where $\overline{\mathcal{C}}$ denotes the closure of $\mathcal{C}$. We recall that, by the Kleiman criterion of ampleness, a line bundle $\mathcal{L}$ is ample if $c_{1}(\mathcal{L})$ is in the interior (in the sense of the topology on $\left.N^{1}(X)\right)$ of the cone $\mathcal{P}$, which we will denote by $\mathcal{P}^{\prime}$.

1. Hard Lefschetz Theorem. In the present section we deal with the classical result of Lefschetz. Our purpose is to understand its impact on the structure of the cohomology ring of a complex projective manifold and on the second cohomology in particular. For the exposition of the result the reader may consult the classical textbook [8] or a recent excellent survey [15].

Let $X$ be a compact complex manifold of dimension $n$. In the present section we discuss some properties of the cohomology ring $\bigoplus_{m \geq 0} H^{m}(X, \mathbf{R})$ which is equipped with the cup product $\cup$. We recall that $b_{m}(X)=\operatorname{dim}_{\mathbf{R}} H^{m}(X, \mathbf{R})$ is the $m$-th Betti number of $X$.

Definition. We will say that $\eta \in H^{2}(X, \mathbf{R})$ satisfies Lefschetz condition if the $k$-th cup product map

$$
\begin{array}{ccc}
L_{k}(\eta): H^{n-k}(X, \mathbf{R}) & \longrightarrow & H^{n+k}(X, \mathbf{R}) \\
\chi & \longrightarrow & \chi \cup \eta^{\cup k}
\end{array}
$$

is an isomorphism for $k=1, \ldots n$ (then we will call it Lefschetz duality).

By abuse, if $\eta=\eta(D)$ is the cohomology class of a Cartier divisor $D$ or, equivalently, the first Chern class $c_{1}(\mathcal{L})$ of the associated line bundle $\mathcal{L}=\mathcal{O}_{X}(D)$ then we will say that $D$ and $\mathcal{L}$ satisfy the Lefschetz condition as well. The above definition is motivated by

HARD Lefschetz TheOREM. If $\mathcal{L}$ is an ample line bundle then it satisfies the Lefschetz condition.

Alternatively, instead of considering the map $L_{k}(\eta)$ we can take $(-1)^{n-k}$-symmetric bilinear pairing

$$
\begin{aligned}
H^{n-k}(X, \mathbf{R}) \times H^{n-k}(X, \mathbf{R}) & \longrightarrow \mathbf{R} \\
(\chi, \nu) & \longrightarrow \chi \cup \nu \cup \eta^{\cup k}
\end{aligned}
$$

In other words, the choice of $\eta$ gives a 2 -form $A_{k}(\eta)$ which lives in $S^{2}\left(H^{n-k}(X, \mathbf{R})^{*}\right)$ or $\bigwedge^{2}\left(H^{n-k}(X, \mathbf{R})^{*}\right)$ - depending on whether $n-k$ is even or odd - and which is nondegenerate if and only if $\eta$ satisfies the $k$-th cup product map is an isomorphism. In a fixed basis the form $A_{k}(\eta)$ can be represented as a symmetric (resp. skew-symmetric) 
$b_{n-k} \times b_{n-k}$ matrix, the coefficients of which are homogeneous of degree $k$ in $\eta$. Therefore, since $A_{k}(\eta)$ is nondegenerate if and only if $\operatorname{det} A_{k}(\eta) \neq 0$, we obtain the following

LEMMA 1.1. Assume that $X$ is a projective manifold. Let $\Delta_{k}=\Delta_{k}(X):=\{\eta \in$ $H^{2}(X, \mathbf{R}): L_{k}(\eta)$ is not an isomorphism $\}$. Then $\Delta_{k}$ is the zero set of a homogeneous polynomial $\delta_{k}=\operatorname{det} A_{k}$ of degree $k b_{n-k}$ (if $n-k$ is odd then $\delta_{k}$ is the square of the pfaffian of $\left.A_{k}\right)$. Moreover, identifying rational homology $\mathrm{H}_{2}(X, \mathbf{Q})$ with rational linear forms on $H^{2}(X, \mathbf{R})$ we have $\delta_{k} \in S^{k b_{n-k}}\left(H_{2}(X, \mathbf{Q})\right)$.

Proof. Lemma follows from the preceding discussion in which the field $\mathbf{R}$ can be replaced by $\mathbf{Q}$ so that $\delta_{k} \in S^{k b_{n-k}}\left(H_{2}(X, \mathbf{Q})\right)$.

The set $\Delta_{k} \subset H^{2}(X, \mathbf{R})$ which is introduced just above will be called the $k$-th Lefschetz discriminant. The immediate consequence of Lefschetz theorem is that for $k=1, \ldots n$ the Lefschetz discriminant $\Delta(X)=\bigcup \Delta_{k}$ does not meet rational points in $\mathcal{P}^{\prime}$.

EXAMPLE. Let $X=\mathbf{C}^{n} / \Gamma$ be a compact complex torus. The cohomology ring of $X$ can be identified with the ring of alternating real forms on $\mathbf{C}^{n}=\mathbf{R}^{2 n}$ that is

$$
\bigoplus_{k} H^{k}(X, \mathbf{R}) \simeq \bigoplus_{k} \bigwedge^{k}\left(\mathbf{R}^{2 n}\right) .
$$

It is easy to verify that a form $\eta$ satisfies Lefschetz condition if and only if $\eta^{\wedge n} \neq 0$, i.e. if it is symplectic. Indeed, this is equivalent that in some basis it can written as $\eta=d x_{1} \wedge d x_{2}+d x_{3} \wedge d x_{4}+\ldots+d x_{2 n-1} \wedge d x_{2 n}$ and thus, for a suitable choice of the complex structure on $\mathbf{R}^{2 n}$, we have $\eta=\sqrt{-1} \cdot\left(d z_{1} \wedge d \bar{z}_{1}+d z_{2} \wedge d \bar{z}_{2}+\ldots+d z_{n} \wedge d \bar{z}_{n}\right)$. In this case the Lefschetz condition is defined by the $n$-th product map, or equivalently for $i=1, \ldots n$ we have $\Delta_{i} \subset \Delta_{n}$.

Definition. A non-zero homology class $\alpha \in H_{2}(X, \mathbf{R})$ is called Lefschetz supported (or simply L-supported) if it divides $\delta_{k}$ for some $k=1, \ldots n$.

By abuse, a line $\mathbf{R} \cdot \alpha \subset H_{2}(X, \mathbf{R})$ or a ray $\mathbf{R}_{\geq 0} \cdot \alpha \subset H_{2}(X, \mathbf{R})$ will be called L-supported if $\alpha$ is L-supported. Moreover, we will say that $\alpha$ is of type $k$ and multiplicity $m$ if it divides $\delta_{k}$ with multiplicity exactly $m$. In this notation we have the following corollary to the previous Lemma.

Corollary 1.2. Let $X$ be a projective manifold. Then the set of L-supported lines in $H^{2}(X, \mathbf{R})$ is finite. More exactly, if $\#(k, m)$ is the number of lines of type $k$ and multiplicity $m$ then for $k=1, \ldots n$ we have

$$
\sum_{m \geq 1} m \cdot \#(k, m) \leq k \cdot b_{n-k} .
$$

The reason to introduce the notion of L-supported homology class is the following consequence of Lefschetz Theorem.

Proposition 1.3. Assume that $X$ is a projective manifold. Let $\alpha$ be an $L$ supported rational homology class in $\mathrm{H}_{2}(X, \mathbf{R})$. Then

(1) the cone $\mathcal{P}$ is not cut by the hyperplane $\alpha^{\perp}$, or equivalently, either $\alpha \cdot \mathcal{P} \geq 0$ or $\alpha \cdot \mathcal{P} \leq 0$

(2) if $\beta \in N_{1}(X)$ is the projection of $\alpha$ along $N^{1}(X)^{\perp}$ (i.e. $\beta$ is the unique class in $N_{1}(X)$ such that $\left.(\beta-\alpha) \cdot N^{1}(X)=0\right)$ then either $\beta \in \overline{\mathcal{C}}$ or $-\beta \in \overline{\mathcal{C}}$.

Proof. Immediate.

REMARK. Lefschetz condition is $\mathbf{R}^{*}$ invariant, that is $\eta \in H^{2}(X, \mathbf{R})$ satisfies it if and only if $a \eta$ does, for any $a \in \mathbf{R} \backslash\{0\}$. Although, for a given complex structure 
the notion of ample cone is only $\mathbf{R}_{>0}^{*}$ invariant, we note however that the topological invariants should not distinguish an ample divisor from its opposite.

Indeed, the topological space of a given complex manifold $X$ supports also its conjugate structure $\bar{X}$ which is associated to the conjugation ${ }^{-}: \mathbf{C} \rightarrow \mathbf{C}$ of the base field. If $\eta \in H^{2}(X, \mathbf{Z})$ is a class of an ample divisor on $X$ then $-\eta$ is the class of an ample divisor on $\bar{X}$.

2. Extremal rays and extremal contractions. The task of the present paper is to apply the formalism which we have introduced in the previous section in the situation appearing in Mori theory of extremal ray contractions. In the present section we use the language and the fundamental results of the Minimal Model Program. For an introduction and an exposition to the program we suggest [5] or more advanced [12]. The cone theorem (smooth case) is explained in [17]. The local analytic version of the contraction theory which we will rely on is in [10].

A contraction is a proper surjective map $\varphi: X \rightarrow Y$ of normal irreducible varieties with connected fibers such that $\varphi_{*} \mathcal{O}_{X}=\mathcal{O}_{Y}$. We assume that a contraction is not an isomorphism. The map $\varphi$ is birational or otherwise $\operatorname{dim} Y<\operatorname{dim} X$, in the latter case we say that $\varphi$ is of fiber type. The exceptional locus $E(\varphi)$ of a birational contraction $\varphi$ is equal to the smallest subset of $X$ such that $\varphi$ is an isomorphism on $X \backslash E(\varphi)$. The contraction $\varphi$ is called Fano-Mori (or just Mori contraction) if the anti-canonical divisor $-K_{X}$ is $\varphi$-ample Q-Cartier divisor. If the map $\varphi$ is birational and $K_{X}=\varphi^{*} K_{Y}$ then we say that $\varphi$ is crepant.

The contraction $\varphi$ yields two maps $\varphi^{*}: H^{2}(Y, \mathbf{Z}) \rightarrow H^{2}(X, \mathbf{Z})$ on cohomology and also $\varphi^{*}: N^{1}(Y) \rightarrow N^{1}(X)$, which we denote similarly. We say that $\varphi$ is elementary if $\operatorname{dim}\left(N^{1}(X) / \varphi^{*} N^{1}(Y)\right)=1$.

If both $X$ and $Y$ are projective then $\varphi^{*}(\mathcal{P}(Y))$ is a face of the cone $\mathcal{P}(X)$. Moreover the contraction $\varphi$ kills (i.e. contracts to points) the holomorphic curves whose classes are perpendicular to the face $\varphi^{*}(\mathcal{P}(Y))$. In particular, an elementary contraction defines a 1 -dimensional face (a ray) in $\mathcal{C}(X)$. The ray is called Mori ray or crepant ray if its contraction is of the respective type.

It is remarkable that in the situation covered by the Minimal Model Program the passage from contractions to rays (or faces) of $\mathcal{C}(X)$ can be reversed. That is, if a projective normal variety $X$ has suitable singularities (e.g. it is smooth) then a "good" face of the cone $\mathcal{C}(X)$ admits a contraction. In particular, if $R \subset \mathcal{C}(X)$ is a ray such that for some $D \in R^{\perp} \cap \mathcal{P}(X), D^{\perp} \cap \mathcal{C}(X)=R$ and either $R \cdot K_{X}<0$ or $R \cdot K_{X}=0$ and $D^{n}>0$, then there exists a contraction $\varphi_{R}: X \rightarrow Y_{R}$ of $R$, where $Y_{R}$ is a projective normal variety. The divisor $D$ is then called a good supporting divisor for $R$ and it is a pullback of an ample divisor from $Y_{R}$. The contraction $\varphi_{R}$ is either Fano-Mori or crepant depending on whether $R \cdot K_{X}<0$ or $R \cdot K_{X}=0$, respectively. This is just a version of

Kawamata-Shokurov Contraction (or Base-Point-Free) Theorem. Assume that $X$ is a projective manifold. Let $D \in P i c X$ be a line bundle such that $[D]$ as well as $\left[a D-K_{X}\right]$ are in $\mathcal{P}(X)$ for some $a>0$. If moreover $\left(a D-K_{X}\right)^{n}>0$ then there exists a contraction $\varphi: X \rightarrow Y$ such that $D=\varphi^{*}\left(D_{Y}\right)$ for some ample divisor $D_{Y} \in P i c Y$.

We note that both Fano-Mori and crepant case are covered by the same theorem. For this reason, in the present paper frequently we do not make any distinction between Fano-Mori or crepant elementary contractions - we will call both extremal contractions which is a slight abuse of the usual notation. 
It turns out that in this range the word "elementary" can be used in a broader sense, i.e. any extremal contraction is elementary also topologically. That is: if $\varphi$ : $X \rightarrow Y$ is an extremal contraction then $H^{2}(X, \mathbf{R}) / \varphi^{*}\left(H^{2}(Y, \mathbf{R})\right) \simeq \mathbf{R}$. This can be derived from results of Kollár and Mori on complex homology, see [14], Theorem 12.1.3. Below I present a similar argument which also yields a result on integral cohomology.

First we need a local version of the Base-Point-Free Theorem. Namely, in the following lemma we will use the set-up and the language explained in [10] pp. 102-106. That is, $X$ is a complex manifold and the contraction $\varphi: X \rightarrow Y$ is now a projective morphism onto a normal analytic space $Y$ with a fixed point $y \in Y$. Shrinking $Y$ if necessary we may assume that it satisfies the assumptions 1.11 of [ibid]. Now we adapt the definitions of $\varphi$-nefness and bigness for the map $\varphi:\left(X, \varphi^{-1}(y)\right) \rightarrow(Y, y)$ as explained at pp. 105-106 of [ibid].

Lemma 2.1. Let $\varphi:\left(X, \varphi^{-1}(y)\right) \rightarrow(Y, y)$ be as above. Assume that $-K_{X}$ is $\varphi$-big and nef with respect to $\{y\}$. If $L \in P i c X$ is a line bundle such that $L \cdot C=0$ for any curve $C \subset \varphi^{-1}(y)$ then there exists an open neighborhood $Y^{\prime}$ of $y$ such that $L$ is trivial on $X^{\prime}=\varphi^{-1}\left(Y^{\prime}\right)$, i.e. $L_{\mid X^{\prime}} \simeq \mathcal{O}_{X^{\prime}}$.

Proof. Because of Theorem 1.3' in [10] $L^{\otimes m}$ is $\varphi$-spanned on $\varphi^{-1}(y)$ for $m \gg 0$. Thus, since $L$ is numerically trivial on $\varphi^{-1}(y)$, we can choose a section of $L^{\otimes m}$ which does not vanish anywhere on $\varphi^{-1}(y)$. Therefore, $L^{\otimes m}$ is trivial in a neighborhood of $\varphi^{-1}(y)$ for $m \gg 0$, so is $L$ itself.

Corollary 2.2. Let $\varphi: X \rightarrow Y$ be a contraction of a projective manifold. Suppose that $-K_{X}$ is $\varphi$-big and nef. Then for any $y \in Y$ and any non-trivial $L \in$ $\left(R^{1} \varphi_{*} \mathcal{O}_{X}^{*}\right)_{y}$ there exists a holomorphic curve $C \subset \varphi^{-1}(y)$ such that $L \cdot C \neq 0$. Thus the group $\left(R^{1} \varphi_{*} \mathcal{O}_{X}^{*}\right)_{y}$ is a finitely generated abelian group with no torsion.

Proof. The first part is just the previous Lemma. To get the second part we use the intersection product $\left(R^{1} \varphi_{*} \mathcal{O}_{X}^{*}\right)_{y} \times H_{2}\left(\varphi^{-1}(y), \mathbf{Z}\right) \rightarrow \mathbf{Z}$ and the first part to get $R^{1} \varphi_{*} \mathcal{O}_{X}^{*} \hookrightarrow \operatorname{Hom}\left(H_{2}\left(\varphi^{-1}(y), \mathbf{Z}\right), \mathbf{Z}\right)$.

Now we are ready to prove the main technical result of this part.

Proposition 2.3. Let $\varphi: X \rightarrow Y$ be a contraction of a projective manifold. Assume that $-K_{X}$ is $\varphi$-big and nef. Then $R^{1} \varphi_{*} \mathbf{Z}_{X}=0$, moreover the cohomology map $\varphi^{*}: H^{2}(Y, \mathbf{Z}) \rightarrow H^{2}(X, \mathbf{Z})$ is injective and the cokernel of the natural inclusion $\operatorname{Pic}(X) / \varphi^{*} \operatorname{Pic}(Y) \hookrightarrow H^{2}(X, \mathbf{Z}) / \varphi^{*} H^{2}(Y, \mathbf{Z})$ is a torsion group.

Proof. Let us consider the direct image of the exponential sequence on $X$ :

$$
0 \longrightarrow R^{1} \varphi_{*} \mathbf{Z}_{X} \longrightarrow R^{1} \varphi_{*} \mathcal{O}_{X} \longrightarrow R^{1} \varphi_{*} \mathcal{O}_{X}^{*} \longrightarrow R^{2} \varphi_{*} Z_{X} \longrightarrow R^{2} \varphi_{*} \mathcal{O}_{X}
$$

Because of the vanishing of $R^{i} \varphi_{*} \mathcal{O}_{X}$ for $i \geq 1$ (see e.g. [10], Theorems 1.2 and 1.2') we get $R^{1} \varphi_{*} \mathbf{Z}_{X}=0$ and isomorphism of sheaves of abelian groups $R^{1} \varphi_{*} \mathcal{O}_{X}^{*} \simeq R^{2} \varphi_{*} \mathbf{Z}_{X}$. We consider a commutative diagram with exact rows coming from Leray spectral sequence and vertical arrows coming from cohomology of the exponential sequence

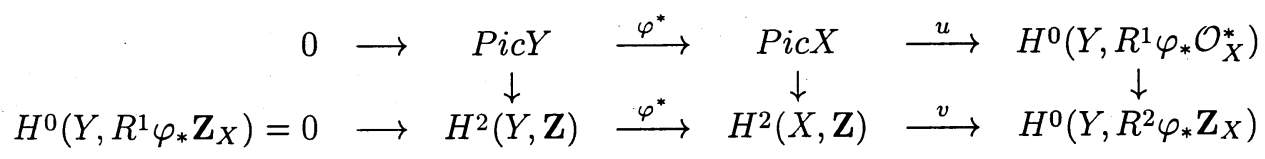

Because of 2.2 the most-to-the-right vertical arrow in an isomorphism of torsionfree abelian groups which will use to identify them. Let $U:=i m(u)$ and $V:=i m(v)$. Then, both $U$ and $V$ are finitely generated abelian with no torsion and because of this identification we can write $U \hookrightarrow V$. 
Let us consider a free abelian group generated by curves contracted by $\varphi$, that is $\mathcal{Z}_{1}(\varphi):=\left\{\sum a_{i} C_{i}: a_{i} \in \mathbf{Z}\right\}$ where $C_{i} \subset X$ are homolorphic curves contracted by $\varphi$, only finite number of $a_{i}$ is non-zero and $\mathcal{Z}_{1}(\varphi)$ has a natural group structure. We have a natural intersection product

$$
H^{0}\left(Y, R^{1} \varphi_{*} \mathcal{O}_{X}^{*}\right) \times \mathcal{Z}_{1}(\varphi) \longrightarrow \mathbf{Z}
$$

In view of 2.2 , for any non-zero $L \in H^{0}\left(Y, R^{1} \varphi_{*} \mathcal{O}_{X}^{*}\right)$ there exists $Z \in \mathcal{Z}_{1}(\varphi)$ such that $L \cdot Z \neq 0$. Therefore the product yields a map $\mathcal{Z}_{1}(\varphi) \rightarrow H o m(V, \mathbf{Z})$ whose cokernel is a torsion group.

Note that to any 1 -cycle $Z \in \mathcal{Z}_{1}(\varphi)$ we can associate its class $[Z]_{X} \in H_{2}(X, \mathbf{Z})$. Now $Z \cdot U=0$ if and only if $[Z]_{X}$ is zero on $c_{1}(\operatorname{Pic} X) \subset H^{2}(X, \mathbf{Z})$. However, since $Z$ is a 1-cycle of holomorphic curves it follows that actually $[Z]_{X}$ vanishes on the whole cohomology $H^{2}(X, \mathbf{Z})$ hence $Z \cdot V=0$. Thus the image of $\mathcal{Z}_{1} \rightarrow H o m(V, \mathbf{Z})$ does not meet non-zero elements in $\operatorname{ker}(H \operatorname{Hom}(V, \mathbf{Z}) \rightarrow \operatorname{Hom}(U, \mathbf{Z})) \simeq \operatorname{Hom}(\operatorname{coker}(U \rightarrow V), \mathbf{Z})$ so the latter group is zero which implies that $\operatorname{coker}(U \hookrightarrow V)$ is a torsion group.

EXAMPLE. The quotient $V / U$ may be actually non-zero (notation as in the proof). Indeed, let $Y$ be an abelian surface and $\varphi: X \rightarrow Y$ a $\mathbf{P}^{1}$-bundle in complex topology which does not come from a rank 2 vector bundle on $Y$. Then $R^{2} \varphi_{*} \mathbf{Z}_{X} \simeq R^{1} \varphi_{*} \mathcal{O}_{X}^{*} \simeq$ $\mathbf{Z}_{Y}, H^{3}(Y, \mathbf{Z}) \simeq \mathbf{Z}^{4}$ and using further terms of the Leray spectral sequence we get a diagram

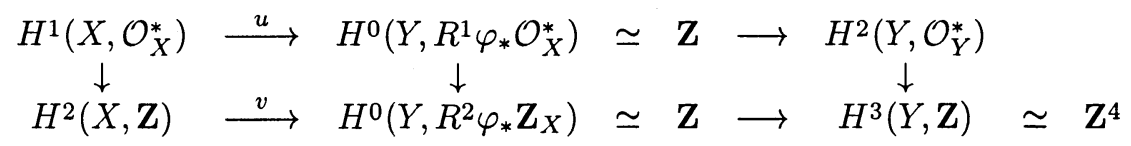

Thus the map $v$ is surjective while $u$ is onto $2 \mathbf{Z} \subset \mathbf{Z}$ since $\mathcal{O}(1)$ on the fiber does not extend to $X$. Finally, let us note that from the above sequence it follows that $V / U \hookrightarrow H^{2}\left(Y, \mathcal{O}_{Y}^{*}\right)$ even if $\varphi$ is not a projective bundle. In fact the group $V / U$ seems to be a good generalization of the invariant $\delta_{r}$ which is defined for projective bundles as in [6].

As a corollary we get the following representability theorem (c.f. Kollár, Mori, [14], Theorem 12.1.3).

THEOREM 2.4. Let $\varphi_{R}: X \rightarrow Y$ be an extremal contraction of a ray $R$ of a projective manifold. Then $R^{\perp}=\varphi_{R}^{*}\left(H^{2}(Y, \mathbf{R})\right)$.

In other words any topological cocycle perpendicular to $R$ is represented by a pullback of a cocycle from the target of the contraction $\varphi_{R}$.

3. L-negligible extremal rays. In this section we assume that $\varphi=\varphi_{R}: X \rightarrow$ $Y$ is an extremal contraction of a ray $R$ on a smooth projective variety of dimension $n$. If $R$ is L-supported then it is noticeable from the topological view point as it gives a strong trace in the cohomology by giving a component of the Lefschetz discriminant. Moreover, we have seen that the number of L-supported rays on $X$ is finite. On the other hand, although from the Mori Cone Theorem in [17] it follows that the extremal rays in the cone $\mathcal{C}(X)$ are discrete, its total number on $X$ may be infinite. (A simple example is a $\mathbf{P}^{2}$ blown up in 9 points so that the resulting surfaces has infinite number of (-1)-curves, see e.g. [5] example 4.6.4)

Therefore one may be tempted to conclude that "a generic extremal ray on a variety $X$ is not L-supported". This gives a motivation for understanding extremal rays which are not L-supported; we will call them $L$-negligible, for short. 
Proposition 3.1. Let $\varphi_{R}: X \rightarrow Y$ be an extremal contraction of a ray $R$ of a projective manifold. If there exists a subset $S \subset X$ such that $2 \operatorname{dim}_{\mathbf{C}} S-\operatorname{dim}_{\mathbf{C}} \varphi(S)=$ $n+m$ for some positive integer $m$ then $R$ is L-supported of type $k:=2 \operatorname{dim}_{\mathrm{C}} S-n$ and multiplicity $\geq m$.

Proof. Let us set $\operatorname{dim}_{\mathbf{C}} S=a, \operatorname{dim}_{\mathbf{C}} \varphi(S)=b-1$ so that $k=2 a-n$ and $m=k-b+1$. Let $[S]_{X} \in H_{2 a}(X, \mathbf{R})$ and $\nu_{S} \in H^{2 n-2 a}(X, \mathbf{R})$ denote the homology and cohomology class of $S$, respectively. Consider a cocycle $\eta_{Y} \in H^{2}(Y, \mathbf{R})$. We claim that $\nu_{S} \cup \varphi^{*}\left(\eta_{Y}\right)^{\cup b}=0$ in $H^{2 n-2 a+2 b}(X, \mathbf{R})$. This is equivalent to show that $[S]_{X} \cap \varphi^{*}\left(\eta_{Y}\right)^{\cup b}=0$ in $H_{2 b-2 a}(X, \mathbf{R})$. Let $i: S \rightarrow X, j: \varphi(S) \rightarrow Y$ be embeddings and $\varphi_{S}: S \rightarrow \varphi(S)$ the restriction of $\varphi$, so that $\varphi \circ i=j \circ \varphi_{S}$. Then

$$
[S]_{X} \cap \varphi^{*}\left(\eta_{Y}\right)^{\cup b}=i_{*}\left([S] \cap i^{*}\left(\varphi^{*}\left(\eta_{Y}\right)\right) \cup b\right)=i_{*}\left([S] \cap \varphi_{S}^{*}\left(j^{*}\left(\eta_{Y}\right)^{\cup b}\right)\right)
$$

but $j^{*}\left(\eta_{Y}\right)^{\cup b} \in H^{2 b}(\varphi(S), \mathbf{R})=0$, because $\operatorname{dim}_{\mathbf{C}} \varphi(S)=b-1$.

Let us choose a cocycle $\chi_{0} \notin R^{\perp}$ and consider an arbitrary $\chi \in H^{2}(X, \mathbf{R})$ which we can write as $\chi=\varphi^{*}\left(\eta_{Y}\right)+t \chi_{0}$ for an appropriate choice of $t \in \mathbf{R}$ and $\eta_{Y} \in$ $H^{2}(Y, \mathbf{R})$. Namely, we set $t=(\alpha \cdot \chi) /\left(\alpha \cdot \chi_{0}\right)$ for a nonzero $\alpha \in R$ and then $\chi-t \chi_{0} \in R^{\perp}$ is in the image of $\varphi^{*}$ by the representability. Then

$$
\chi^{\cup k} \cup \nu_{S}=t^{k-b+1}\left(\begin{array}{c}
k \\
b-1
\end{array}\right) \cdot \eta_{Y}^{b-1} \cup \chi_{0}^{k-b+1} \cup \nu_{S}+\ldots+t^{k} \chi_{0}^{k} \cup \nu_{S}
$$

Therefore, if we write the matrix $A_{k}(\chi)$ of the Lefschetz duality $L_{k}(\chi)$ (notation from Section 1) in a basis $\left\{\nu_{0}:=\nu_{S}, \nu_{1}, \ldots\right\}$ of the cohomology $H^{n-k}(X, \mathbf{R})$ then the first column is divisible by $t^{m}$. Thus $\delta_{k}$ is divisible by $t^{m}$ which concludes the proof of the proposition.

REMARK. If in the situation of the above proposition $2 \operatorname{dim}_{\mathbf{C}} S-\operatorname{dim}_{\mathbf{C}} \varphi(S)=n$ and $R$ is not L-supported, then the class $\nu_{S}$ of $S$ is $\varphi^{*}\left(\eta_{Y}\right)$-primitive (notation as in the above proof, $\varphi^{*}\left(\eta_{Y}\right)$ satisfies Lefschetz condition), that is $\nu_{S} \cup \varphi^{*}\left(\eta_{Y}\right)^{(k+1)}=$ 0 ; moreover the class $\nu_{F}$ of a general fiber of $\varphi_{\mid S}$ is equal to some multiplicity of $\nu_{S} \cup \varphi^{*}\left(\eta_{Y}\right)^{k}$ (so, up to some multiplicity, it is Lefschetz dual to $\nu_{S}$ ). All this follows from arguments similar to these used above in the proof of 3.1.

The above result should be compared with the following property of extremal contractions, see [9] Theorem 0.4, [7] Lemma 2.5 and [23] Theorem 1.1.

THEOREM 3.2. Let $\varphi=\varphi_{R}: X \rightarrow Y$ be an extremal contraction of a ray $R$ and let $S \subset E(\varphi)$ be an irreducible component of the exceptional locus of $\varphi$. We define the length $l_{S}(R)$ of $R$ at $S l_{S}(R):=\min \left\{-K_{X} \cdot[C]\right\}$ where $C$ is a rational curve passing through a general point of $S$ and $[C] \in R$. Then we have

$$
2 \operatorname{dim}_{\mathbf{C}} S-\operatorname{dim}_{\mathbf{C}} \varphi(S) \geq n+l_{S}(R)-1 \text {. }
$$

The inequality appearing in the above theorem is sometimes referred to as fiberlocus inequality. The bounds provided by the fiber-locus inequality and the inequality from 3.2 give a narrow space for L-negligible extremal contractions. If $R$ is crepant then $l_{S}(R)=0$ and $2 \operatorname{dim}_{\mathbf{C}} S-\operatorname{dim}_{\mathbf{C}} \varphi(S)$ is either $n-1$ or $n$.

If however $R$ is a Mori ray then the exceptional locus of $\varphi_{R}$ is covered by rational curves such that $-K_{X} \cdot[C]=1$. Moreover, for any component $S$ of $E(\varphi)$ we have $2 \operatorname{dim}_{\mathrm{C}} S-\operatorname{dim}_{\mathrm{C}} \varphi(S)=n$. As the consequence, Mori L-negligible rays can be pretty well described. Thus for the rest of this section we assume that $R$ is a Mori L-negligible 
ray. A similar situation (when the fiber-locus inequality becomes an equality) was considered in Lemma 1.1 of [4].

Lemma 3.3. Let $\varphi: X \rightarrow Y$ be a contraction of a Mori L-negligible ray. For an irreducible component $S \subset E(\varphi)$ let $r:=\operatorname{codim}_{X} S=\operatorname{dim}_{\mathbf{C}} S-\operatorname{dim}_{\mathbf{C}} \varphi(S)$. If $F$ is an irreducible component of a fiber of $\varphi_{\mid S}$ such that $\operatorname{dim}_{\mathbf{C}} F=r$ then its normalization $f: \tilde{F} \rightarrow F \subset S$ is isomorphic to a projective space $\tilde{F} \simeq \mathbf{P}^{r}$ and $f^{*} \mathcal{O}_{X}\left(-K_{X}\right) \simeq \mathcal{O}(1)$.

The dimension of the exceptional locus and the dimension of fibers of a Fano-Mori contraction of a L-negligible ray are tied up very closely so that in small dimensions (or small codimensions) fibers of such contractions are small and thus were thoroughly studied. The subsequent structure theorem summarizes some of the known properties of such contractions. We refer the reader to [3] for an exhausting description of FanoMori contraction with fibers of dimension $\leq 2$.

THEOREM 3.4. Let $\varphi=\varphi_{R}: X \rightarrow Y$ be a Fano-Mori contraction of an $L$ negligible ray on a smooth projective variety. Let $E=E(\varphi)$ denote the exceptional locus of $\varphi$ and let $Z:=\varphi(E) \subset Y$ be the exceptional locus of $\varphi^{-1}$. If $Z_{k}:=\{y \in Y$ : $\left.\operatorname{dim}_{\mathbf{C}} f^{-1}(y) \geq k\right\} \subset Z$ then $\operatorname{dim}_{\mathbf{C}} Z_{k} \leq n-2 k$. Moreover,

(i) if $\operatorname{dim}_{\mathbf{C}} E=n-1$ then $E$ is an irreducible divisor, $\operatorname{dim}_{\mathbf{C}} Z=n-2$; outside the set $Z_{2}$ both $Y$ and $Z$ are smooth and outside of the set $Z_{3}$ the map $\varphi^{-1}$ is a blow-up of $Y$ along $Z$.

(ii) if $\operatorname{dim}_{\mathbf{C}} E \leq n-2$ then $Z=Z_{2}$ and outside a set $Z^{\prime} \subset Z$, $\operatorname{dim}_{\mathbf{C}} Z^{\prime} \leq n-5$, the map $f^{-1}$ is a small resolution of a family of Veronese cone singularities. That is, for any $y \in Z \backslash Z^{\prime}$ there exists a neighborhood $U$ biholomorphic to $\Delta^{n-4} \times V^{4}$ where $\Delta^{n-4}$ is a complex disc of dimension $n-4$ and $V^{4}$ is a neighborhood of the vertex of the cone over Veronese embedding $\mathbf{P}^{1} \times \mathbf{P}^{2} \hookrightarrow \mathbf{P}^{5}$. In particular, outside $f^{-1}\left(Z^{\prime}\right)$ any non-trivial fiber of $\varphi$ is $\mathbf{P}^{2}$ with the normal $\mathcal{O}(-1)^{\oplus 2} \oplus \mathcal{O}^{n-4}$.

Proof. The estimate on the dimension of $Z_{k}$ is obvious. The irreducibility of $E$ in (i) as well the description of $\varphi$ outside of $\varphi^{-1}\left(Z_{2}\right)$ is in [1], whereas a general blow-up statement is in [3]. The description of $\varphi$ in (ii) follows easily from [11].

For some of the applications of extremal rays which are mentioned in the introduction it is convenient to know the scheme parameterizing rational curves from an extremal ray. Below, we sketch the construction of such an object. For an overview on the theory of $\operatorname{Hilb}(X), \operatorname{Chow}(X)$ and $\operatorname{Hom}\left(\mathbf{P}^{1}, X\right)$ - and how they can be used to parameterize rational curves - we refer the reader to [13], Chapter II.1. Rational curves were firstly discussed in this context by Mori in [16] who proved their existence in extremal rays in [17].

Let us recall that an extremal rational curve in a Mori ray $R$ is a rational curve $C \subset X$ with a normalization $f: \mathbf{P}^{1} \rightarrow C \subset X$ such that $[C] \in R$ and its degree $\operatorname{deg}\left(f^{*}\left(-K_{X}\right)\right)=-K_{X} \cdot[C]$ is minimal among the curves from $R$. In particular, if $R$ is L-negligible then $-K_{X} \cdot[C]=1$.

Let us take a component $\mathcal{M} \subset \operatorname{Hom}\left(\mathbf{P}^{1}, X\right)$ which contains the class of $f$. We consider the image of $\mathcal{M}$ under the natural map $\operatorname{Hom}\left(\mathbf{P}^{1}, X\right) \rightarrow \operatorname{Chow}(X)$ which sends $f \in \operatorname{Hom}\left(\mathbf{P}^{1}, X\right)$ to the class of $f\left(\mathbf{P}^{1}\right)$. The image of this map (after a normalization) is the geometric quotient $\mathcal{M} / G$ where $G=\operatorname{Aut}\left(\mathbf{P}^{1}\right)$ acts on $\mathcal{M}$ by composition $G \times \mathcal{M} \ni(g, f) \mapsto f \circ g^{-1} \in \mathcal{M}$. Now we take the fiber product $\mathcal{W}:=\mathcal{M} \times{ }_{G} \mathbf{P}^{1}$ which has a natural projection $q: \mathcal{W} \rightarrow \mathcal{M} / G$ and the evaluation $e v: \mathcal{W} \rightarrow X$ such that $e v(f, t)=f(t)$. The quotient $\mathcal{M} / G$ is a complete scheme (compact analytic space) and it coincides with the normalization of the appropriate component of $\operatorname{Chow}(X)$ which contains $C=f\left(\mathbf{P}^{1}\right)$. Moreover, at a generic point it is the same as the appropriate component of $\operatorname{Hilb}(X)$. The dimension of $\mathcal{M} / G$ is bounded from below by 
Riemann-Roch:

$$
\operatorname{dim}_{\mathbf{C}} \mathcal{M} / G \geq-K_{C} \cdot[C]+n-3 .
$$

We will say that $C$ has deformations of the expected dimension if the the above inequality becomes an equality for any component $\mathcal{M} \subset \operatorname{Hom}\left(\mathbf{P}^{1}, X\right)$ which contains $[f]$.

The line of arguments presented above is due to Mori [16]. We note also the following useful observation

Mori Breaking Lemma. For any two different points $x_{1}, x_{2} \in X$ the intersection $q\left(e v^{-1}\left(x_{1}\right)\right) \cap q\left(e v^{-1}\left(x_{2}\right)\right)$ is finite.

For L-negligible rays we have the following

LEMma 3.5. Assume that $R$ is an L-negligible Mori ray of a manifold $X$. If $C \subset X$ is an extremal rational curve from $R$ then its deformations are the of expected dimension.

Proof. We use the notation introduced above. By Breaking Lemma the dimension of fibers of $\left(\varphi_{R}\right)_{\mid e v(\mathcal{W})}$ is at least by 1 bigger than the dimension of the respective fibers of $e v$. Indeed, for any $x \in e v(\mathcal{W})$ the map $e v$ on $q^{-1}\left(q\left(e v^{-1}(x)\right)\right)$ is finite-to-one outside of $e v^{-1}(x)$. Therefore

$$
\operatorname{dim}_{\mathbf{C}} e v(\mathcal{W})-\operatorname{dim}_{\mathbf{C}} \varphi_{R}(e v(\mathcal{W})) \geq \operatorname{dim}_{\mathbf{C}} \mathcal{W}-\operatorname{dim}_{\mathbf{C}} e v(\mathcal{W})+1
$$

Since $\operatorname{dim}_{\mathbf{C}} \mathcal{W}=\operatorname{dim}_{\mathbf{C}} \mathcal{M} / G+1$, in view of 3.1 we have

$$
n \geq 2 \operatorname{dim}_{\mathbf{C}} e v(\mathcal{W})-\operatorname{dim}_{\mathbf{C}} \varphi_{R}(e v(\mathcal{W})) \geq \operatorname{dim}_{\mathbf{C}} \mathcal{M} / G+2
$$

which is what we want.

REMARK. Even in the case of a divisorial contraction, c.f. 3.4, although the exceptional locus of $\varphi$ is irreducible, the scheme of extremal rational curves may be reducible. In fact, in 6.9 of [3] we describe a three component Hilbert scheme of extremal rational curves of a divisorial contraction of a smooth 4-fold with a reducible fiber which is a degenerate quadric $\mathbf{P}^{2} \cup \mathbf{P}^{2}$. On the other hand [11] gives an example of a small contraction of a 4 -fold with a disconnected exceptional locus which is a union of arbitrary (finite) number of disjoint copies of $\mathbf{P}^{2}$.

We note however that Lemma 3.5 refers to any component of the scheme of extremal rational curves. Moreover, from the above proof of 3.5 it follows that the Remark following Proposition 3.1 as well as the conclusion of Lemma 3.3 holds also when we set $S=e v(\mathcal{W})$. Indeed, in the quoted example from [3] the special fiber of the divisorial contraction consists of two $\mathbf{P}^{2}$.

Before stating a corollary to the above lemma let us recall that given a curve $C \subset X$ we say that the class of $C$ remains holomorphic for small deformations of $X$ if for any smooth family $\pi: \mathcal{X} \rightarrow \Delta^{1}$ of compact complex manifolds over a disc $\Delta^{1}$ such that $\pi^{-1}(0) \simeq X$ and any $t$ sufficiently small there exists a holomorphic curve $C_{t} \subset \pi^{-1}(t)$ such that $\left[C_{t}\right]=[C]$ under the natural identification $H_{2}\left(\pi^{-1}(t), \mathbf{Z}\right)=$ $H_{2}(X, \mathbf{Z})$.

COROLlary 3.6. The class of an extremal rational curve in an L-negligible Mori ray on a projective manifold $X$ remains holomorphic for small deformations of $X$.

In fact the Corollary is a consequence of 3.5 if we use the following observation (c.f. [2]). 
Lemma 3.7. Let $C \subset X$ be a rational curve in a compact complex manifold. If the dimension of deformations of $C$ inside $X$ is of the expected dimension then the class of $[C]$ remains holomorphic for small deformations of $X$.

Proof. The dimension of deformations of $C$ inside $\mathcal{X}$ is bounded from below by $\operatorname{dim}_{C} \mathcal{X}-K_{\mathcal{X}} \cdot[C]-3=n-K_{X} \cdot[C]-2$ so it is bigger than the dimension of deformations of $C$ in $X$. Therefore the curve $C$ in $\mathcal{X}$ has to move out from $\pi^{-1}(0)$ to the neighboring fibers.

In the conclusion let me make some comments on the geometry of L-negligible rays in the context of Gromov-Witten invariants. As before, let us choose an extremal rational curve $C$ and an irreducible component $\mathcal{M} \subset \operatorname{Hom}\left(\mathbf{P}^{1}, X\right)$ containing the normalization of $C$. For any positive $k$ we consider the product $\mathcal{W}_{k}:=$ $\mathcal{M} \times{ }_{G}\left(\mathbf{P}^{1}\right)^{\times k}$ where $G=\operatorname{Aut}\left(\mathbf{P}^{1}\right)$ acts on the $k$-th product $\left(\mathbf{P}^{1}\right)^{\times k}$ coordinatewise $G \times\left(\mathbf{P}^{1}\right)^{\times k} \ni\left(g,\left(t_{1}, \ldots t_{k}\right)\right) \mapsto\left(g\left(t_{1}\right), \ldots g\left(t_{k}\right)\right) \in\left(\mathbf{P}^{1}\right)^{\times k}$. On $\mathcal{W}_{k}$ we have the natural projection $q: \mathcal{W}_{k} \rightarrow \mathcal{M} / G$ and the evaluation $e v_{k}: \mathcal{W}_{k} \rightarrow X^{\times k}$ with $e v_{k}\left(f, t_{1}, \ldots t_{k}\right)=\left(f\left(t_{1}\right), \ldots f\left(t_{k}\right)\right)$. We recall that since $C$ is extremal it follows that $\mathcal{M} / G$ is compact and $e v_{k}$ is proper.

We are interested in case $k=2$. In this case however, because of the breaking lemma, the map $e v_{2}$ does not have positive dimensional fibers outside of the diagonal of $X \times X$. Thus, if $S_{2} \subset X^{\times 2}$ is the image of $e v_{2}$, then $\operatorname{dim}_{\mathbf{C}} S_{2}=\operatorname{dim}_{\mathbf{C}} \mathcal{M}-1 \geq$ $n-K_{X} \cdot[C]-1$. Suppose that $-K_{X} \cdot[C]=1$ and $\mathcal{M}$ is of the expected dimension, then $\operatorname{dim}_{\mathrm{C}} S_{2}=n$. Let $p_{i}: X \times X \rightarrow X$ be the projection on the $i$-factor. Given two cohomology classes $\alpha_{i} \in H^{a_{i}}(X, \mathbf{Z})$ with $a_{1}+a_{2}=2 n$ we consider the bilinear map

$$
\left(\alpha_{1}, \alpha_{2}\right)_{\mathcal{M}}:=\left(p_{1}^{*}\left(\alpha_{1}\right) \cup p_{2}^{*}\left(\alpha_{2}\right)\right) \cdot\left[S_{2}\right] .
$$

Let $S=p_{i}\left(S_{2}\right)=e v\left(\mathcal{M} \times{ }_{G} \mathbf{P}^{1}\right)$ be the locus of curves from $\mathcal{M}$ in $X$ and set $s=\operatorname{dim}_{\mathbf{C}} S$. Moreover for $x \in S$ let $S_{x}:=e v\left(q^{-1}\left(q\left(e v^{-1}(x)\right)\right)\right)$. We note that if for some $i=1,2$ we have $a_{i}>2 s$ then $\alpha_{i} \cap p_{i *}\left(S^{2}\right)=0$ and thus $\left(\alpha_{1}, \alpha_{2}\right)_{\mathcal{M}}=0$. Thus the product can be non-zero only if $2 s \geq a_{i} \geq 2 n-2 s$ for $i=1,2$. On the other hand if $a_{1}=2 s$ and $a_{2}=2 n-2 s$ then we find out that

$$
\left(\alpha_{1}, \alpha_{2}\right)_{\mathcal{M}}=\left(\alpha_{1} \cdot[S]\right) \cdot\left(\alpha_{2} \cdot\left[S_{x}\right]\right)
$$

where $x \in S$ is general.

In particular, if $R$ is a L-negligible extremal ray then the above discussion applies to any extremal rational curve in $R$. Moreover, because of the remarks following the proofs of 3.1 and 3.5 the Lefschetz duality $L_{k}$ behaves nice with respect to $(,)_{\mathcal{M}}$. That is, for any two classes $\alpha_{1}, \alpha_{2} \in H^{2 n-2 s}(X, \mathbf{R}), k=2 s-n$ and $\eta \in R^{\perp}$ satisfying Lefschetz condition we have

$$
\left(\alpha_{1}, L_{k}(\eta)\left(\alpha_{2}\right)\right)_{\mathcal{M}}=\left(L_{k}(\eta)\left(\alpha_{1}\right), \alpha_{2}\right)_{\mathcal{M}} .
$$

Similar arguments were used in [19] to compute Gromov-Witten invariants of some special extremal rational curves. I must admit however that in the discussion presented above I ignored verification of the assumptions which are usually asked in the symplectic set-up to make the computation of Gromov-Witten invariants legible. This can be done in low dimensional cases by Theorem (3.4).

Appendix: remarks and questions. First let us note that if $X$ is not projective then the notion of the Lefschetz discriminant may become void. For example: a Hopf surface. 
We noted that Hard Lefschetz Theorem implies that $\Delta$ does not meet the interior $\mathcal{P}^{\prime}$ of the nef cone in rational points. Can $\Delta$ meet $\mathcal{P}^{\prime}$ at non-rational points?

The bound on the number of L-supported rays obtained by adding degrees of $\Delta_{i}$ is probably not the best because subsequent loci $\Delta_{i}$ are related one to another. What would be the best bound for the number of L-supported rays?

The definitions and the results from Section 1 can be introduced for complex cohomology ring $H^{*}(X, \mathbf{C})$. In particular we can introduce complex Lefschetz discriminants $\Delta_{\mathbf{C}}(X)$ and respective L-supported rays. It is clear that the "unbalanced" components of the Hodge decomposition of $H^{2}(X, \mathbf{C})$, i.e. $H^{0,2}(X)$ and $H^{2,0}(X)$ are contained in $\Delta_{\mathbf{C}}(X)$. Moreover from the results of Section 2 it follows that if $R$ is an extremal L-supported ray on $X$ then $R_{\mathbf{C}}^{\perp} \supset H^{2,0}(X) \oplus H^{0,2}(X)$. Indeed, if $\varphi_{R}: X \rightarrow Y_{R}$ is the contraction of $R$ then $H^{2}\left(X, \mathcal{O}_{X}\right)=\varphi_{R}^{*} H^{2}\left(Y, \mathcal{O}_{Y}\right) \subset R_{\mathrm{C}}^{\perp}$ and obviously $R_{\mathbf{C}}^{\perp}$ is invariant of the complex conjugation of $H^{2}(X, \mathbf{C})$

Suppose that $X^{\prime}$ is another complex structure which defines the respective Hodge decomposition of $H^{2}(X, \mathbf{C})$. Is it true that still $H^{2,0}\left(X^{\prime}\right) \oplus H^{0,2}\left(X^{\prime}\right) \subset R_{\mathbf{C}}^{\perp}$ ? What if $X^{\prime}$ is obtained by a complex deformation of $X$ ? The results of [24] may suggest that the answer for the second question is positive.

The inequality $2 \operatorname{dim}_{\mathbf{C}} S-\operatorname{dim}_{\mathbf{C}} \varphi_{R}(S)>n$, which appears in Proposition 3.1, is a sufficient condition for an extremal ray $R$ to be L-supported. Let us note however that it destroys the Lefschetz duality $(\dagger \dagger)$ on an even cohomology group of $X$. I have been unable to find an example of an extremal ray such that the inequality $2 \operatorname{dim}_{\mathbf{C}} S-\operatorname{dim}_{\mathbf{C}} \varphi_{R}(S) \leq n$ is satisfied for any complex subset $S$ of $X$ and despite of this it is L-supported (e.g. the Lefschetz duality fails on odd cohomology). However it is hard to expect that the condition from Proposition 3.1 on an extremal ray to be L-supported is a necessary one.

\section{REFERENCES}

[1] ANDo, T., On extremal rays of the higher dimensional varieties, Invent. Math. 81 (1985), 347-357.

[2] Andreatta, M., Peternell, Тн., On the limits of manifolds with nef canonical bundle, preprint (1996).

[3] Andreatta, M., Wiśniewski J. A., On contractions of smooth varieties, J. Alg. Geom., 7 (1998), 253-312.

[4] Andreatta, M., Ballico, E., Wiśniewski, J. A., Two theorems on elementary contractions, Math. Ann., 297 (1993), 191-198.

[5] Clemens, H., Kollár, J., MoRI, S., Higher dimensional complex geometry, Asterisque, 166 (1988).

[6] Elencwajg, G., The Brauer group in complex geometry, in Brauer groups in ring theory and algebraic geometry, Antwerp 1981, Springer Lect. Notes in Math 917 (1982), 222-230.

[7] Fujita, T., On polarized manifolds whose adjoint bundles are not numerically effective, Adv. Stud. Pure Math. 10 (1987), 167-178.

[8] Griffiths, Ph., Harris, J., Principles of Algebraic Geometry, Wiley Interscience Publication, 1978.

[9] Ionescu, P., Generalized adjunction and applications, Math. Proc. Cambridge Math. Soc., 99 (1988), 457-472.

[10] KaWamata, Y., Crepant blowing-up of 3-dimensional canonical singularities and ist application to degeneration of surfaces, Ann. Math., 127 (1988), 93-163.

[11] KaWAmata, Y., Small contractions of four dimensional algebraic manifolds, Math. Ann., 284 (1989), 595-600. 
[12] Kawamata, Y., Matsuda, K., Matsuki, K., Introduction to the Minimal Model Program in Algebraic Geometry, Sendai, Adv. Studies in Pure Math., 10, Kinokuniya-North-Holland 1987, 283-360.

[13] Kollár, J., Rational Curves on Algebraic Varieties, Springer Verlag, Ergebnisse der Math., 32, 1995 .

[14] Kollár, J., Mori, S., Classification of three-dimensional fips, J. American Math. Soc., 5 (1992), 533-703.

[15] Looijenga, E., Cohomology and intersection homology of algebraic varieties, in Complex Alg. Geom. ed. Kollár, IAS/Park City Math Series, 3, AMS-IAS 1997, 221-263.

[16] MoRI, S., Projective manifolds with ample tangent bundle, Ann. Math., 110 (1979), 593-606.

[17] MORI, S, Threefolds whose canonical bundles are not numerically effective, Ann. Math., 116 (1982), 133-176.

[18] McDuff, D., Salamon, D, J-holomorphic curves and quantum cohomology, Univ. Lect. Series 6 AMS 1994.

[19] Paoletti, R., On symplectic invariants of algebraic varieties coming from crepant contractions, preprint (1996)

[20] RUAN, Y., Symplectic topology and extremal rays, Geom. Funct. Analysis, 3 (1992) 279-291.

[21] Wilson, P.M.H., Symplectic deformations of Calabi-Yau threefolds, J. Diff. Geometry., 45 (1997), 611-637.

[22] WIŚNIEWSKI J. A., Length of extremal rays and generalized adjunction, Math. Zeit., 200 (1990), 409-427.

[23] WiśnieWSKI, J. A., On contractions of extremal rays of Fano manifolds, Jounal für die reine und angew. Mathematik, 417 (1991), 141-157.

[24] Wiśniewski, J. A., On deformation of nef values, Duke Math. J., 64 (1991), 325-332. 
